\title{
Generalized Anti Fuzzy Ideals in Near-Rings
}

Tariq Anwar ${ }^{1 *}$, Mammuhad Naeem ${ }^{2}$ and Saleem Abdullah ${ }^{3}$

'Department of Mathematics, Quaid i Azam University Islamabad Pakistan; tariqanwar79@yahoo.co.in ²Deanship of Preparatory Year, Umm Al Qurrs University, Makkah, Saudi Arabia; naeemtakeer@yahoo.com 32Department of Mathematics, Quaid i Azam University Islamabad Pakistan; saleemabdullah81@yahoo.com

\begin{abstract}
In this paper, we generalize anti-fuzzy ideals of near-rings, introduce the concept of $(<,<\vee \Upsilon)$-fuzzy ideals, prime $(<,<\vee \Upsilon)$ fuzzy ideals, semiprime $(<,<\vee \Upsilon)$-fuzzy ideals of near-rings and discuss some properties of such ideals.
\end{abstract}

Keywords: Near-ring, Fuzzy Set, Anti Fuzzy Point, $(<,<\vee \Upsilon)$-Fuzzy Ideals

AMS Classification: 16Y60, 13E05, 03G25.

\section{Introduction}

The notion of near-ring was first introduced by Dickson and Leonard in 1905 [1]. We note that the ideals of near-rings play a central role in the structure theory, however, they do not in general coincide with the usual ring ideals of a ring.

The concept of a fuzzy set was first introduced by Zadeh [2]. Fuzzy set theory has been shown to be a useful tool to describe situations in which the data are imprecise or vague. Fuzzy sets handle such situations by attributing a degree to which a certain object belongs to a set. The fuzzy algebraic structures play a major role in mathematics with wide applications in many other branches such as theoretical physics, computer sciences, control engineering, information sciences, coding theory, topological spaces, logic, set theory, group theory, real analysis, measure theory etc. The notions of fuzzy subnear-ring and ideal were first introduced by Abou-Zaid in [3]. In Hong et al. [4] and Kim et al. [5] introduced the concept of fuzzy ideals of near-ring and obtained some useful results in near-rings.

In Biswas [6] introduced the concept of anti-fuzzy subgroups of groups, Kim and Jun studied the notion of anti-fuzzy R-subgroups of near-ring in [7], and Kim et al. studied the notion of anti-fuzzy ideals in near-rings in [8].
In [9] Shabir and Rehman introduced the concept of anti-fuzzy left (right, lateral) ideals, anti-fuzzy quasiideals, anti-fuzzy bi-ideals and anti-fuzzy generalized bi-ideals in ternary semigroups. In [10] Jun et al. introduced the concept of generalized anti-fuzzy bi-ideals in ordered semigroups. The reader is also refereed to [11-15].

In this paper, we generalize anti-fuzzy ideals of nearrings, introduce the concept of $(<,<\vee \Upsilon)$-fuzzy ideals, prime $(<,<\vee \Upsilon)$-fuzzy ideals and semiprime $(<,<\vee \Upsilon)$-fuzzy ideals of near-rings by using the idea in [16] of "beside to" relation $(<)$ and the "non-quasi-coincident" relation $(\Upsilon)$ between an anti-fuzzy point and a fuzzy set and discuss some properties of such ideals.

\section{Preliminaries}

Definition 1. [17] A near-ring $N$ is an algebraic system $(N,+,$.$) consisting of a non-empty set N$ together with two binary operations addition "+" and multiplication "'." such that

(1) $(N,+)$ is a group,

(2) $(N,$.$) is a semigroup,$

(3) $(x+y) \cdot z=x \cdot z+y \cdot z$ for all $x, y, z \in N$.

*Corresponding author:

Tariq Anwar (tariqanwar79@yahoo.co.in) 
We will use the word "near-ring" to mean "right distributive near-ring" and $x y$ instead of $x$. $y$. Note that $0 x=0$ and $(-x) y=x y$ but in general $0 x \neq 0$ for some $x \in$ $N$. The sets $N_{0}=\{x \in N \mid x 0 \in 0\}$ and $N_{c}=\{x \in N \mid x 0 \in x\}=$ $\{x \in N \mid x y=x$, for all $y \in N\}$ are called zero symmetric and constant part of $N$, respectively. $N_{0}$ and $N_{c}$ are near-rings. $N$ is caled zero symmetric (constant) if $N=N_{0}\left(N=N_{c}\right)$.

Definition 2. [17] Let $(N,+,$.$) be a near-ring. A subset A$ of $N$ is said to be an ideal of $N$ if

(1) $(A,+)$ is a normal subgroup of $(N,+)$,

(2) $A N \subseteq A$,

(3) $n_{1}\left(n_{2}+a\right)-n_{1} n_{2} \in A$, for all $a \in A$ and $n_{1}, n_{2} \in N$.

If $A$ satisfies (1) and (2), then it is called a right ideal of $N$. If $A$ satisfies (1) and (3), then it is called a left ideal of $N$. In what follows, $N$ will denote right distributive near-ring, unless otherwise specified.

If $S$ and $T$ are subsets of $N$, we denote the set $\{s t: s \in S, t \in T)$ by $S T$. An ideal $P$ of $N$ is called a prime ideal if for any ideal $I$ and $J$ of $N, I J \subseteq P$ implies either $I \subseteq P$ or $J \subseteq P$. An ideal $I$ of $N$ is called a semi-prime ideal if for any ideal $J$ of $N, J^{2} \subseteq I$ implies that $J \subseteq I$.

A fuzzy subset $f$ of a universe set $X$ is a function from $X$ into the unit closed interval $[0,1]$, i.e. $f: X \rightarrow[0,1]$. For any two fuzzy subsets $f$ and $g$ of $X$. The product $f \circ g$ is defind by

$$
(f \circ g)(x)=\left\{\begin{array}{c}
\sup _{x=a b}\{\min (f(a), g(b))\} \\
0 \text { otherwise }
\end{array}\right.
$$

$f \leq g$ means that, for all $x \in X, f(x) \leq g(x)$. Through out this paper max, min, sup, and inf are used for maximum, minimum, sup remum and inf imum respectively. Define $f^{c}, f \cap g, f \cup g$ as follows: $f^{c}(x)=1-f(x),(f \cap g)$ $(x)=\min \{f(x), g(x)\},(f \cup g)(x)=\max \{f(x), g(x)\}$, for all $x \in X . f^{c}$ is called the complement of $f, f \cap g$ is called the intersection of $f$ and $g$, and $f \cup g$ is called the union of $f$ and $g$. Let $A$ be a subset of $X$ the characteristic function of $A$ is the function $C_{A}$ of $X$ into $\{0,1\}$ defined by

$$
C_{A}(x)= \begin{cases}1 & \text { if } x \in A \\ 0 & \text { if } x \notin A .\end{cases}
$$

For $s \in 0,1]$, the set $X_{f}^{s}=\{x \in X f(x) \leq s\}$ is called lower $s$-level cut of $f$.
Definition 3. [16] A fuzzy set $f$ in $X$ of the form $f(y)=\left\{\begin{aligned}s \in 0,1) & \text { if } y=x, \\ 1 & \text { if } y \neq x,\end{aligned}\right.$ is called an anti-fuzzy point with support $x$ and value s and is denoted by $x_{s}$.

A fuzzy set $f$ in $X$ is said to be non unit if there exists $x \in X$ such that $f(x)<1$.

Definition 4. [16] An anti-fuzzy point $x_{s}$ is said to beside to (resp. be non-quasi coincident with) a fuzzy set $f$, denoted by $x_{s}<f\left(\right.$ resp. $\left.x_{s} \Upsilon \mathrm{r} f\right)$, if $f(x) \leq s($ resp. $f(x)+s<1)$. We say that $<$ (resp. $\Upsilon$ ) is a beside to (resp. non-quasi coincident with) relation between anti-fuzzy points and fuzzy sets.

If $x_{s}<f$ or $x_{s} \Upsilon f$ we say that $x_{s}<\vee \Upsilon f$ and $x_{s}<f$ (resp. $x_{s} \Upsilon \bar{\Upsilon} f, x_{s}<\vee \Upsilon f$ ) means that $x_{s}<f$ (resp. $x_{s} \Upsilon f, x_{s}<\vee \Upsilon f$ ) does not hold.

Definition 5. [8] A fuzzy subset $f$ of a near-ring $N$ is called an anti-fuzzy subnear-ring of $N$ if for all $x, y \in N$ :

(1) $f(x-y) \leq \max \{f(x), f(y)\}$ and

(2) $f(x y) \leq \max \{f(x), f(y)\}$.

Definition 6. [8] A fuzzy subset $f$ of a near-ring $N$ is called an anti-fuzzy ideal of $N$ if for all $x, y, a \in N$,

(1) $f(x-y) \leq \max \{f(x), f(y)\}$,

(2) $f(y+x-y) \leq f(x)$,

(3) $f(x y) \leq f(x)$ and

(4) $f(x(y+a)-x y) \leq f(a)$.

If $f$ satisfies (1), (2) and (3), then it is called an anti-fuzzy right ideal of $N$. If $f$ satisfies (1), (2) and (4), then it is called an anti-fuzzy left ideal of $N$.

Definition 7. For any two anti-fuzzy ideals $f$ and $g$ of $N$. The product $f \circ g$ is defined by

$$
(f \circ g)(x)= \begin{cases}\inf _{x=a b}\{\max (f(a), g(b))\}, \\ 1 & \text { otherwise. }\end{cases}
$$

Proposition 1. Let $A$ be a non-empty subset of a near-ring $N$ and $f$ be a fuzzy set defined by

$$
f(x)= \begin{cases}s_{1} & \text { if } x \in A, \\ s_{2} \text { otherwise }\end{cases}
$$

where $1 \geq s_{2}>s_{1} \geq 0$, then $f$ is an anti-fuzzy left(right) ideal of $N$ iff $A$ is a left(right) ideal of $N$. 


\section{3. $(<,<\vee r)$-Fuzzy Ideals}

In this section we define $(<,<\vee \Upsilon)$-fuzzy ideals of near-rings and investigate some properties of these ideals.

Definition 8. A fuzzy set $f$ of $N$ is called $a(<,<)$ - fuzzy subnear-ring of $N$ if for all $x, y \in N$, and $\left.s_{1}, s_{2} \in 0,1\right)$.

(a) $x_{s_{1}}<f, y_{s_{2}}<f \Rightarrow(x-y)_{\max \left\{s_{1}, s_{2}\right\}}<f$,

(b) $x_{s_{1}}<f, y_{s_{2}}<f \Rightarrow(x y)_{\max \left\{s_{1}, s_{2}\right\}}<f$.

Definition 9. A fuzzy set $f$ of $N$ is called $a(<,<)$ - fuzzy right(resp, left) ideal of $N$ if for all $x, y \in N$, and $\left.s_{1}, s_{2} \in 0,1\right)$.

(a) $x_{s_{1}}<f, y_{s_{2}}<f \Rightarrow(x-y)_{\max \left\{s_{1}, s_{2}\right\}}<f$,

(b) $x_{s_{1}}<f, y \in N \Rightarrow(y+x-y)_{s_{1}}<f$,

(c) $x_{s_{1}}<f, y \in N \Rightarrow(x y)_{s_{1}}<f$ (resp. If $a_{s_{1}}<f, x, y \in$ $\left.N \Rightarrow(x(y+a)-x y)_{s_{1}}<f\right)$.

A fuzzy set which is a $(<,<)$-fuzzy right and left ideal of $N$ is called a $(<,<)$-fuzzy ideal of $N$.

TheOrem 1: A fuzzy set $f$ of $N$ is a $(<,<)$ - fuzzy subnearring of $N$ if and only if it is an anti-fuzzy subnear-ring of $N$.

Proof: Let $f$ be a $(<,<)$-fuzzy subnear-ring of $N$. (a) Let $x, y \in N$, and $\left.s_{1}, s_{2} \in 0,1\right)$. If $f(x)=1$ or $f(y)=1$, then $f(x-y) \leq 1=\max \{f(x), f(y)\}$. If $f(x)=s_{1} \neq 1$ or, $f(y)=s_{2} \neq 1$ then $x_{s_{1}}, y_{s_{2}}<f$ and by assumption $(x-y)_{\max \left\{s_{1}, s_{2}\right\}}<f$. This implies that $f(x-y) \leq \max \left\{s_{1}, s_{2}\right\}=\max \{f(x), f(y)\}$. (b) Let $x, y \in N$ and $\left.s_{1}, s_{2} \in 0,1\right)$. If $f(x)=1$ or $f(y)=1$, then $f(x y) \leq 1=\max \{f(x), f(y)\}$. If $f(x)=s_{1} \neq 1$ or $f(y)=s_{2} \neq 1$, then $x_{s_{1}}, y_{s_{2}}<f$ and by assumption $(x y)_{\max \left\{s_{1}, s_{2}\right\}}<f$. This implies that $f(x y) \leq \max \left\{s_{1}, s_{2}\right\}=\max \{f(x), f(y)\}$.

Conversely, suppose that $f$ is an anti-fuzzy subnear-ring of N. $y_{s_{2}}<f$

(a) If $x_{s_{1}}<f$, and $y_{s_{2}}<f$ for $\left.s_{1}, s_{2} \in 0,1\right)$. Then, $f(x) \leq s_{1}$ and $f(y) \leq s_{2}$, by assumption we have $f(x-y) \leq \max$ $\{f(x), f(y)\} \leq \max \left\{s_{1}, s_{2}\right\}$. Thus, $(x-y)_{\max \left\{s_{1}, s_{2}\right\}}<f$. (b) Let $x_{s_{1}}<f, y_{s_{2}}<f$ for $\left.s_{1}, s_{2} \in 0,1\right)$. Then $f(x) \leq s_{1}$ and $f(y) \leq s_{2}$, we have $f(x y) \leq \max \{f(x), f(y)\} \leq \max \left\{s_{1}, s_{2}\right\}$. Thus, $(x y)_{\max \left\{s_{1}, s_{2}\right\}}<f$ and hence $\mathrm{f}$ is a $(<,<)$-fuzzy subnearring of $N$.

THeOREM 2: A fuzzy set $f$ of $N$ is a $(<,<)$ - fuzzy right ideal of $N$ if and only if it is an anti-fuzzy right ideal of $N$.

Proof: Let $f$ be a $(<,<)$-fuzzy right ideal of $N$. (a) Let $x, y \in N$ and $\left.s_{1}, s_{2} \in 0,1\right)$. Then $f(x-y) \leq \max \left\{s_{1}, s_{2}\right\}=\max$ $\{f(x), f(y)\}$, by Theorem 1. (b) Let suppose that there exist $x, y \in N$ such that $f(y+x-y)>f(x)$. Choose $s \in 0,1)$ such that $f(y+x-y)>s>f(x)$. So, $x_{s_{1}}<f$ : but $(y+x-y)_{s_{1}} \overline{<} f$, a contradiction. Hence, $f(y+x-y) \leq f(x)$. (c) Suppose that there exist $x, y \in N$ such that $f(x y)>f(x)$. Choose $s \in 0,1)$ such that $f(x y)>s>f(x)$. So, $x_{s_{1}}<f$ but $(x y)_{s_{1}} \overline{<} f$, a contradiction. Hence, $f(x y) \leq f(x)$. and hence $f$ is an anti-fuzzy right ideal of $N$.

Conversely, suppose that $f$ is an anti-fuzzy right ideal of $N$. (a) Let $x_{s_{1}}<f, y_{s_{2}}<f$ for some $\left.s_{1}, s_{2} \in 0,1\right)$. Then, $(x-y)_{\max \left\{s_{1}, s_{2}\right\}}<f$, by Theorem 1. (b) Now, for $y \in N$ and $x_{s_{1}}<f$, since $f(y+x-y) \leq f(x) \leq s$. Thus, $(y+x-y)_{s_{1}}<f$. (c) Now, for $y \in N$ and $x_{s_{1}}<f$, by assumption since $f(x y) \leq f(x) \leq s_{1}$. Thus, $(x y)_{s_{1}}<f$. Hence, $f$ is a $(<,<)$-fuzzy right ideal of $N$.

THeOREM 3: A fuzzy set $f$ of $N$ is a $(<,<)$-fuzzy left ideal of $N$ if and only if it is an anti-fuzzy left ideal of $N$.

Proof: Let $f$ be a $(<,<)$-fuzzy left ideal of $N$. (a) Let $x, y \in N$. Then $f(x-y) \leq \max \left\{s_{1}, s_{2}\right\}=\max \{f(x), f(y)\}$, by Theorem 1. (b) If $x, y \in N$. Then $f(y+x-y) \leq f(x)$, by Theorem 2. (c) Suppose that there exist $x, y, a \in N$ such that $f(a)=s$. This implies that $a_{s}<f$ and by assumption $(x(y+a)-x y)_{s}<f$, so $f(x(y+a)-x y) \leq s=f(a)$.

Conversely, suppose that $f$ is an anti-fuzzy left ideal of $N$. (a) Let $x_{s_{1}}<f, y_{s_{2}}<f$, for $\left.s_{1}, s_{2} \in 0,1\right)$. Then, $(x-y)_{\max \left\{s_{1}, s_{2}\right\}}<f$, by Theorem 1. (b) If $y \in N$ and $x_{s_{1}}<f$, Then, $(y+x-y)_{s_{1}}<f$, by Theorem 2. (c) Now consider for $x, y, a \in N$ and $a_{s_{1}}<f$, by assumption since $f(x(y+a)$ $x y) \leq f(a) \leq s_{1}$. Thus, $(x(y+a)-x y)_{s_{1}}<f$. Hence, $f$ is a $(<,<)$-fuzzy left ideal of $N$.

Definition 10. A fuzzy set $f$ of $N$ is called $a(<,<\vee \Upsilon)$ - fuzzy subnear-ring of $N$ if for all $x, y \in N$ and $\left.s_{1}, s_{2} \in 0,1\right)$.

(a) If $x_{s_{1}}<f, y_{s_{2}}<f \Rightarrow(x-y)_{\max \left\{s_{1}, s_{2}\right\}}<\vee \Upsilon f$

(b) If $x_{s_{1}}<f, y_{s_{2}}<f \Rightarrow(x y)_{\max \left\{s_{1}, s_{2}\right\}}<\vee \Upsilon f$

Definition 11. A fuzzy set $f$ of $N$ is called $a(<,<\vee \Upsilon)$ - fuzzy right (resp. left) ideal of $N$ if for all $x, y \in N$, and $\left.s_{1}, s_{2} \in 0,1\right)$.

(a) If $x_{s_{1}}<f, y_{s_{2}}<f \Rightarrow(x-y)_{\max \left\{s_{1}, s_{2}\right\}}<\vee \Upsilon f$,

(b) If $x_{s_{1}}<f, y \in N \Rightarrow(y+x-y)_{s_{1}}<\vee \Upsilon f$, 
(c) If $x_{s_{1}}<f, y \in N \Rightarrow(x y)_{s_{1}}<\vee \Upsilon f$ (resp. If $a_{s_{1}}<f, x, y$ $\left.\in N \Rightarrow(x(y+a)-x y)_{s_{1}}<\vee \Upsilon f\right)$.

A fuzzy set which is a $(<,<)$-fuzzy right and left ideal of $N$ is called a $(<,<)$-fuzzy ideal of $N$.

Example 1. [18] Consider the set $N=\{0, a, b, c\}$ be Klein four group. Define multiplication in $\mathrm{N}$ as follow:

\begin{tabular}{c|llll}
+ & 0 & $a$ & $b$ & $c$ \\
\hline 0 & 0 & $a$ & $b$ & $c$ \\
$a$ & $a$ & 0 & $c$ & $b$ \\
$b$ & $b$ & $c$ & 0 & $a$ \\
$c$ & $c$ & $b$ & $a$ & 0
\end{tabular}

\begin{tabular}{l|llll}
$\cdot$ & 0 & $a$ & $b$ & $c$ \\
\hline 0 & 0 & 0 & 0 & 0 \\
$a$ & $a$ & $a$ & $a$ & $a$ \\
$b$ & 0 & 0 & 0 & $b$ \\
$c$ & $a$ & $a$ & $a$ & $c$
\end{tabular}

Then, clearly $(N,+,$.$) is a near-ring. Now, let define f$ as: $f(0)=0.2, f(a)=f(c)=0.6, f(b)=0.1$. Then, by routine calculation $f$ is a $(<,<)$-fuzzy sub near-ring of $N$, but is not an anti-fuzzy sub near-ring of $N$, because $f(b-b)=f(0)=0.2$ and $\max \{f(b), f(b)\}=0.1$, that is $f(b-b) \max \{f(b), f(b)\}$.

Example 2. Consider Example 1, since $I=\{0, a\}$ is an ideal of $N$. Let $f$ be fuzzy subset of $N$ such that $f(0)=0, f(a)=0.4$. For this fuzzy subset $f$, let $0.7 \in 0,1)$. Then $f$ is $(<,<\vee \Upsilon)$-fuzzy ideal of $N$.

Example 3. Consider the near-ring $(N,+,$.$) , where N$ is the set of all integers. Define a fuzzy subset $f$ of $N$ by,

$$
f= \begin{cases}0.2 & \text { if } x \in\langle 4\rangle \\ 0.1 & \text { if } x \in\langle 2\rangle-\langle 4\rangle \\ 0.5 & \text { otherwise }\end{cases}
$$

One can easily check that $f$ is a $(<,<)$-fuzzy right ideal of $N$. THEOREM 4: Let $f$ be a $(<,<\vee \Upsilon)$-fuzzy subnear-ring of $N$. Then, the set $N_{1}=\{x \in N: f(x)<1\} \neq \varnothing$ is a subnear-ring of $N$.

Proof: Let $x, y \in N_{\mathrm{r}}$. Then $f(x)<1$ and $f(y)<1$. Assume that $x-y \notin N_{1}$ then $f(x-y)=1$. Then $x_{f(x)}<f$ and $y_{f(y)}<f$ but $(x-y)_{\{\max f(x), f(y)\}} \overline{<\vee \Upsilon} f$, a contradiction. Therefore, $x-y \in N_{1}$. Similarly, $x y \in N_{1}$

TheOREM 5: Let fbe $a(<,<\vee \Upsilon)$-fuzzy right ideal of $N$. Then, the set $N_{1}=\{x \in N: f(x)<1\} \neq \varnothing$ is a right ideal of $N$.

\section{ProOF:}

(a) Let $x, y \in N_{1}$. Then $x-y \in N_{1}$, by Theorem 4 .

(b) If $x \in N_{1}$ and $y \in N$. Then, $f(x)<1$. Suppose that $y+x-y \notin N_{1}$ then $f(y+x-y)=1$. Then, $x_{f(x)}<f$ but $(y+x-y)_{f(x)} \overline{<\vee \Upsilon} f$, a contradiction. Therefore, $y+x-y \in N_{1}$. (c) Let $x \in N_{1}$ and $y \in N$. Then, $f(x)<1$. Suppose that $x y \notin N_{1}$ then $f(x y)=1$. Then, $x_{f(x)}<f$ but $(x y)_{f(x)} \overline{<\vee \Upsilon} f$, a contradiction. Therefore, $x y \in N_{1}$. This completes the proof.

TheOREM 6: Let $f$ be $a(<,<\vee \Upsilon)$-fuzzy left ideal of $N$. Then, the set $N_{1}=\{x \in N: f(x)<1\} \neq \varnothing$ is a left ideal of $N$.

\section{Proof:}

(a) Let $x, y \in N_{1}$ then $x-y \in N_{1}$ by Theorem 4 .

(b) If $x \in N_{1}$ and $y \in N$. Then, $(y+x-y) \in N_{1}$ by Theorem 5 .

(c) Let $a \in N_{1}$ and $x, y \in N$. Then, $f(a)<1$. Suppose that $(x(y+a)-x y) \notin N_{1}$ then $f(x(y+a)-x y)=1$. Then, $a_{f(a)}<f$ but $(x(y+a)-x y) \overline{<\vee \Upsilon} f$, a contradiction. Thus, $f(x(y+a)-x y)<1$. Therefore, $(x(y+a)-x y) \in N_{1}$. This completes the proof.

THEOREM 7: Let $M$ be a subnear-ring of $N$ and $f$ a fuzzy set of $N$ such that:

(i) $f(x) \leq 0.5$ for $x \in M$,

(ii) $f(x)=1$ otherwise

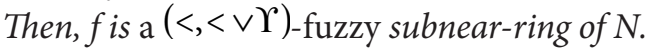

\section{Proof:}

(a) Let $x, y \in N$ and $\left.s_{1}, s_{2} \in 0,1\right)$ be such that $x_{s_{1}}, y_{s_{2}}<f$. Then, $f(x) \leq s_{1}$, and $f(y) \leq s_{2}$. Thus, $x, y \in M$ and so $x-y \in M$, that is $f(x-y) \leq 0.5$. If $\max \left\{s_{1}, s_{2}\right\} \geq 0.5$, then $f(x-y) \leq 0.5 \leq \max \left\{s_{1}, s_{2}\right\}$. Hence, $(x-y)_{\max \left\{s_{1}, s_{2}\right\}}$ $<f$. If $\max \left\{s_{1}, s_{2}\right\}<0.5$, then $f(x-y)+\max \left\{s_{1}\right.$, $\left.s_{2}\right\}<0.5+0.5=1$. Hence, $(x-y)_{\max \left\{s_{1}, s_{2}\right\}} \Upsilon f$. Therefore, $(x-y)_{\max \left\{s_{1}, s_{2}\right\}}<\vee \Upsilon f$.

(b) Now, let $x, y \in N$ and $\left.s_{1}, s_{2} \in 0,1\right)$ be such that $x_{s_{1}}, y_{s_{2}}$ $<f$. Then, $f(x) \leq s_{1}$ and $f(y) \leq s_{2}$. Thus, $x, y \in M$ and so $x y \in M$, that is $f(x y) \leq 0.5$. If $\max \left\{s_{1}, s_{2}\right\} \geq 0.5$, then $f(x-y) \leq 0.5 \leq \max \left\{s_{1}, s_{2}\right\}$. Hence, $(x y)_{\max \left\{s_{1}, s_{2}\right\}}<f$. If $\max \left\{s_{1}, s_{2}\right\}<0.5$, then $f(x y)+\max \left\{s_{1}, s_{2}\right\}<0.5+$ $0.5=1$. Hence, $(x y)_{\max \left\{s_{1}, s_{2}\right\}} \Upsilon f$. Therefore, $(x y)_{\max \left\{s_{1}, s_{2}\right\}}$ $<\vee \Upsilon f$. Hence, $f$ is a $(<,<\vee \Upsilon)$-fuzzy subnear-ring of $N$.

THEOREM 8: Let I be a right ideal of $N$ and $f$ a fuzzy set of $N$ such that:

(i) $f(x) \leq 0.5$ for $x \in I$,

(ii) $f(x)=1$ otherwise.

Then, $f$ is $a(<,<\vee \Upsilon)-$ fuzzy right ideal of $N$. 


\section{Proof:}

(a) Let $x, y \in N$ and $\left.s_{1}, s_{2} \in 0,1\right)$ be such that $x_{s_{1}}, y_{s_{2}}<f$. Then $(x-y)_{\max \left\{s_{1}, s_{2}\right\}}<\vee \Upsilon f f$ proved in Theorem 7 .

(b) Now, let $x, y \in N$ and $s \in 0,1)$ be such that $x_{s}<f$. Then, $f(x) \leq s$. Thus, $x \in I$ and $y \in N$, and so $y+x-$ $y \in I$, since $I$ is right ideal of $N$. Consequently $f(y+$ $x-y) \leq 0.5$. If $s \geq 0.5$, then $f(y+x-y) \leq 0.5 \leq s$ and so $(y+x-y)_{s}<f$. If $s<0.5$, then $f(y+x-y)+s<$ $0.5+0.5=1$ and so $(y+x-y) \Upsilon \mathrm{s} f$. Therefore, $(y+x-y)_{s}<\vee \Upsilon f$.

(c) Now, let $x, y \in N$ and $s \in 0,1)$ be such that $x_{s}<f$. Then, $f(x) \leq s$. Thus, $x \in I$ and $y \in N$, and so $x y \in I$, since $I$ is right ideal of $N$. Consequently $f(x y) \leq 0.5$. If $s \geq 0.5$, then $f(x y) \leq 0.5 \leq s$ and so $(x y)_{s}<f$. If $s<0.5$, then $f(x y)+s<0.5+0.5=1$ and so $(x y)_{5} \vee \Upsilon f$. Therefore, $(x y)_{s}<\vee \Upsilon f$. Hence, $f$ is a $(<,<)$-fuzzy right ideal of $N$. This completes the proof.

THEOREM 9: Let I be a left ideal of $N$ and $f$ a fuzzy set of $N$ such that:

(i) $f(x) \leq 0.5$ for $x \in I$,

(ii) $f(x)=1$ otherwise.

Then, $f$ is a $(<,<\vee \Upsilon)$ - fuzzy left ideal of $N$.

\section{Proof:}

(a) Let $x, y \in N$ and $\left.s_{1}, s_{2} \in 0,1\right)$ be such that $x_{s_{1}}, y_{s_{2}}<f$. Then, $(x-y)_{\max \left\{s_{1}, s_{2}\right\}}<\vee \Upsilon f$, by Theorem 7 .

(b) Now, let $x, y \in N$ and $s \in 0,1)$ be such that $x_{s}<f$. Then, $(y+x-y)_{s}<\vee \Upsilon f$, by Theorem 8 .

(c) Now, let $x, y, a \in N$ and $s \in 0,1)$ be such that $a_{s} \in A$. Then, $f(a) \leq s$. Thus, $a \in I$ and $x, y \in N$, and so $x(y+a)-x y \in I$, since $I$ is right ideal of $N$. Consequently $f(x(y+a)-x y) \leq 0.5$. If $s \geq 0.5$, then $f(x(y+a)-x y) \leq 0.5 \leq s$ and so $(x(y+a)-x y)_{s}<f$. If $s<0.5$, then $f(x(y+a)-x y)+s<0.5+0.5=1$ and so $(x(y+a)-x y)_{s} \Upsilon f$. Therefore, $(x(y+a)-$ $x y)_{s}<\vee \Upsilon f$. This completes the proof.

LEMMA 1: For a fuzzy set $f$ in $N$, the following conditions are equivalent:

(a) $x_{s_{1}}$ and $y_{s_{2}}<f \Rightarrow(x-y)_{\max \left\{s_{1}, s_{2}\right\}}<\vee \Upsilon f$,

(b) $f(x-y) \leq \max \{f(x), f(y), 0.5\}$, for all $x, y \in N$ and $\left.s_{1}, s_{2} \in 0,1\right)$.

\section{ProOF:}

$(a) \Rightarrow(b)$ : Suppose that there exist $x, y \in N$ such that $f(x-y)>\max \{f(x), f(y), 0.5\}$. If $\max \{f(x), f(y)\}>0.5$, then $f(x-y)>\max \{f(x), f(y)\}$. Let choose $\left.s_{1} \in 0,1\right)$ such that $f(x-y)>s_{1}>\max \{f(x), f(y)\}$. This implies $x_{s_{1}}$ and $y_{s_{1}}<f$ but $(x-y)_{s_{1}} \overline{<\vee \Upsilon} f$, which is a contradiction. Now, we consider the case, if $\max \{f(x), f(y)\} \leq 0.5$, then $f(x-y)>0.5$. Thus $x_{0.5}<f$ and $y_{0.5}<f$ but $(x-$ $y)_{0.5} \overline{<\vee \Upsilon} f$, which is a contradiction. Hence, we conclude that $f(x-y) \leq \max \{f(x), f(y), 0.5\}$.

$(b) \Rightarrow(a)$ : Let $x_{s_{1}}, y_{s_{2}}<f$. Then, since $f(x-y) \leq \max \{f$ $(x), f(y), 0.5\}$, so $f(x-y) \leq \max \left\{s_{1}, s_{2}, 0.5\right\}$. Hence, we obtain $f(x-y) \leq \max \left\{s_{1}, s_{2},\right\}$, for $\max \left\{s_{1}, s_{2}\right\} \geq 0.5$. Also, we obtain $f(x-y) \leq 0.5$, for $\max \left\{s_{1}, s_{2}\right\}<0.5$. Therefore, we have $(x-y)_{\max \left\{s_{1}, s_{2}\right\}}<\vee \Upsilon r f$.

LEMMA 2: For a fuzzy set $f$ in $N$, the following conditions are equivalent:

(a) $x_{s_{1}}$ and $y_{s_{2}}<f \Rightarrow(x y)_{\max \left\{s_{1}, s_{2}\right\}}<\vee \Upsilon f$,

(b) $f(x y) \leq \max \{f(x), f(y), 0.5\}$ for all $x, y \in N$ and $\left.s_{1}, s_{2} \in 0,1\right)$.

\section{Proof:}

$(a) \Rightarrow(b)$ : Suppose that there exist $x, y \in N$ such that $f(x y)>\max \{f(x), f(y), 0.5\}$. If $\max \{f(x), f(y)\}>0.5$, then $f(x y)>\max \{f(x), f(y)\}$. Let choose $\left.s_{1} \in 0,1\right)$ such that $f(x y)>s_{1}>\max \{f(x), f(y)\}$. This implies $x_{s_{1}}$ and $y_{s_{1}}<f$ but $(x y)_{s_{1}} \overline{<\vee \Upsilon} f$, which is a contradiction. Now, we consider the case, if $\max \{f(x), f(y)\} \leq 0.5$, then $f(x y)>0.5$. Thus $x_{0.5}$ and $y_{0.5}<f$ but $(x y)_{0.5} \overline{<\vee \Upsilon} f$, which is a contradiction. Hence, we conclude that $f(x y) \leq \max \{f(x), f(y), 0.5\}$.

$(b) \Rightarrow(a)$ : Let $x_{s_{1}}, y_{s_{2}}<f$ for some $\left.s_{1}, s_{2} \in 0,1\right)$. By assumption we have $f(x y) \leq \max \{f(x), f(y), 0.5\}$, so $f(x y)$ $\leq \max \left\{s_{1}, s_{2}, 0.5\right\}$. Hence, we obtain $f(x y) \leq \max \left\{s_{1}\right.$, $\left.s_{2},\right\}$, for $\max \left\{s_{1}, s_{2}\right\} \geq 0.5$. Also, we obtain $f(x y) \leq 0.5$, for $\max \left\{s_{1}, s_{2}\right\}<0.5$. Therefore, we have $(x y)_{\max \left\{\left\{_{1}, s_{2}\right\}\right.}<$ $\vee \Upsilon f$.

LEMмA 3: For a fuzzy set $f$ in $N$, the following conditions are equivalent for all $x, y \in N$ and $s \in 0,1)$ :

(a) $y \in N$ and $x_{s}<f \Rightarrow(x y)_{s}<\vee \Upsilon f$,

(b) $f(x y) \leq \max \{f(x), 0.5\}$. 


\section{Proof:}

$(a) \Rightarrow(b)$ : Let $x, y \in N$ and suppose that $f(x y)>$ $\max \{f(x), 0.5\}$. If $f(x)>0.5$, then $f(x y)>f(x)$. Choose $s \in 0,1)$ such that $f(x y)>s>f(x)$. This implies that $x_{s}<f$ but $(x y)_{s} \overline{<\vee \Upsilon} f$, which is a contradiction. Therefore, $f(x y) \leq \max \{f(x), 0.5\}$. Now, if $f(x) \leq 0.5$, then $f(x y)>0.5$. This implies that $y_{0.5}<f$ but $(x y)_{0.5} \overline{<\vee \Upsilon} f$, again a contradiction. Therefore, $f(x y) \leq \max \{f(x), 0.5\}$. $(b) \Rightarrow(a)$ : Let $x, y \in N$ and $s \in 0,1)$ such that $x_{s}<f$. Then, $f(x) \leq s$. Since $f(x y) \leq \max \{f(x), 0.5\}$, so $f(x y) \leq$ $\max \{s, 0.5\}$. If $s \geq 0.5$, then $f(x y) \leq s$ and so $(x y)_{s}<f$. If $s<0.5$, then $f(x y) \leq 0.5$ and so $f(x y)+s<0.5+0.5=1$. Hence, $(x y)_{s} \Upsilon f$. Thus, $(x y)_{s}<\vee \Upsilon f$. This completes the proof.

LEMMA 4: For a fuzzy set $f$ in $N$, the following conditions are equivalent for all $x, y \in N$ and $s \in 0,1)$ :

(a) $y \in N$ and $x_{s}<f \Rightarrow(y+x-y)_{s}<\vee \Upsilon f$,

(b) $f(y+x-y) \leq \max \{f(x), 0.5\}$.

\section{Proof:}

$(a) \Rightarrow(b)$ : Let $x, y \in N$ and suppose that $f(y+x-y)>$ $\max \{f(x), 0.5\}$. If $f(x)>0.5$, then $f(y+x-y)>f(x)$. Choose $s \in 0,1)$ such that $f(y+x-y)>s>f(x)$. This implies that $x_{s}<f$ but $(y+x-y)_{s} \overline{<\vee \Upsilon} f$, which is a contradiction. Therefore, $f(y+x-y) \leq \max \{f(x), 0.5\}$. Now, if $f(x) \leq 0.5$, then $f(y+x-y)>0.5$. This implies that $x_{0.5}<f$ but $(y+x-y)_{0.5} \overline{<\vee \Upsilon} f$, which is a contradiction. Therefore, $f(y+x-y) \leq \max \{f(x), 0.5\}$.

$(b) \Rightarrow(a)$ : Let $x, y \in N$ and $s \in 0,1)$ such that $x_{s}<f$. Then, $f(x) \leq s$. Since $f(y+x-y) \leq \max \{f(x), 0.5\}$, so $f(y+x-y) \leq \max \{s, 0.5\}$. If $s \geq 0.5$, then $f(y+x-y) \leq s$ and so $(y+x-y)_{s}<f$. If $s<0.5$, then $f(y+x-y) \leq 0.5$ and so $f(y+x-y)+s$ $<0.5+0.5=1$. Hence, $(y+x-y)_{s} \Upsilon f$. Thus, $(y+x-$ $y)_{s}<\vee \Upsilon f$. This completes the proof.

LEMMA 5: For a fuzzy set $f$ in $N$, the following conditions are equivalent for all $x, y, a \in N$ and $s \in 0,1)$ :

(a) $x, y, a \in N$ and $a_{s}<f \Rightarrow(x(y+a)-x y)_{s}<\vee \Upsilon f$,

(b) $f(x(y+a)-x y) \leq \max \{f(a), 0.5\}$.

\section{ProOF:}

$(a) \Rightarrow(b)$ : Let $x, y \in N$ and suppose that $f(x(y+a)-$ $x y)>\max \{f(a), 0.5\}$. If $f(a)>0.5$, then $f(x(y+a)-$ $x y)>f(a)$. Choose $s \in 0,1)$ such that $f(x(y+a)-$ $x y)>s>f(a)$. This implies that $a_{s}<f$ but $(x(y+a)$ $-x y)_{s} \overline{<\vee \Upsilon} f$, which is a contradiction. Therefore, $f(x$ $(y+a)-x y) \leq \max \{f(a), 0.5\}$. Now, $f(a) \leq 0.5$, then $f(x$ $(y+a)-x y)>0.5$. This implies that $a_{0.5}<f$ but $(x(y+$ $a)-x y)_{0.5} \overline{<\vee \Upsilon} f$ which is a contradiction. Therefore, $f(x(y+a)-x y) \leq \max \{f(a), 0.5\}$.

$(b) \Rightarrow(a)$ : Let $x, y, a \in N$ and $s \in 0,1)$ such that $a_{s}<f$. Then, $f(a) \leq s$. Since $f(x(y+a)-x y) \leq \max \{f(a), 0.5\}$, so $f(x(y+a)-x y) \leq \max \{s, 0.5\}$. If $s \geq 0.5$, then $f(x$ $(y+a)-x y) \leq s$ and so $(x(y+a)-x y)_{s}<f$. If $s<0.5$, then $f(x(y+a)-x y) \leq 0.5$ and so $f(x(y+a)-x y)+$ $s<0.5+0.5=1$. Hence, $(x(y+a)-x y)_{s} \Upsilon f$. Thus, $(x(y$ $+a)-x y)_{s}<\vee \Upsilon f$. This completes the proof.

THEOREM 10: Let $f$ be a fuzzy subset in $N$. Then, $f$ is a $(<,<\vee \Upsilon)$-fuzzy sub-nearing if and only if for all $x, y \in N$ :

(a) $f(x-y) \leq \max \{f(x), f(y), 0.5\}$,

(b) $f(x y) \leq \max \{f(x), f(y), 0.5\}$.

Proof: This follows from Lemma 2, and Lemma 1.

THEOREM 11: Let $f$ be a fuzzy subset in N. Then, $f$ is a $(<,<\vee \Upsilon)-$ fuzzy right ideal if and only if for all $x, y \in N$ :

(a) $f(x-y) \leq \max \{f(x), f(y), 0.5\}$,

(b) $f(y+x-y) \leq \max \{f(x), 0.5\}$,

(c) $f(x y) \leq \max \{f(x), 0.5\}$.

Proof: This follows from Lemma 1, Lemma 3 and Lemma 4.

THeOREM 12: Let $f$ be a fuzzy subset in $N$. Then, $f$ is a $(<,<\vee \Upsilon)$-fuzzy left ideal if and only if for all $x, y, a \in N$ :

(a) $f(x-y) \leq \max \{f(x), f(y), 0.5\}$,

(b) $f(y+x-y) \leq \max \{f(x), 0.5\}$,

(c) $f(x(y+a)-x y) \leq \max \{f(a), 0.5\}$.

Proof: This follows from Lemma 1, Lemma 3 and Lemma 5.

TheOrem 13: Any $(<,<\vee \Upsilon)_{\text {-fuzzy sub-near-ring of } N \text { such }}$ that $f(x)>0.5$ for all $x \in N$ is a $(<,<)$-fuzzy sub-near-ring of $N$.

Proof: Let $f$ be a $(<,<\vee \Upsilon)$-fuzzy sub-near-ring of $N$ such 
that $f(x)>0.5$ for all $x \in N$.

(a) Let $x, y \in N$ and $\left.s_{1}, s_{2} \in 0,1\right)$ such that $x_{s_{1}}, y_{s_{2}}<f$.

Then, $f(x) \leq s_{1}$ and $f(y) \leq s_{2}$, we have

$$
\begin{aligned}
f(x-y) \leq & \max \{f(x), f(y) 0.5\} \\
& =\max \{f(x), f(y)\} \leq \max \left\{s_{1}, s_{2}\right\} .
\end{aligned}
$$

This implies that $(x-y)_{\max \left\{s_{1}, s_{2}\right\}}<f$.

(b) Let $x, y \in N$ and $\left.s_{1}, s_{2} \in 0,1\right)$ such that $x_{s_{1}}, y_{s_{2}}<f$.

Then, $f(x) \leq s_{1}$ and $f(y) \leq s_{2}$, we have

$$
\begin{aligned}
f(x y) \leq & \max \{f(x), f(y), 0.5\} \\
& =\max \{f(x), f(y)\} \leq \max \left\{s_{1}, s_{2}\right\} .
\end{aligned}
$$

This implies that $(x y)_{\max \left\{s_{1}, s_{2}\right\}}<f$. Hence $f$ is a $(<,<)$-fuzzy sub-near-ring of $N$.

THEOREM 14: Any $(<,<\vee \Upsilon)$-fuzzy right ideal of $N$ such that $f(x)>0.5$ for all $x \in N$ is a $(<,<)$-fuzzy right ideal of $N$.

Proof: Let $f$ be a $(<,<)$-fuzzy left ideal of $N$ such that $f(x)>0.5$ for all $x \in N$.

(a) Let $x, y \in N$ and $\left.s_{1}, s_{2} \in 0,1\right)$ such that $x_{s_{1}}, y_{s_{2}}<f$. Then, $(x-y)_{\max }\left\{s_{1}, s_{2}\right\}<f$, by Theorem 13 .

(b) Now, let $x, y \in N$ and $s \in 0,1)$ such that $x_{s}<f$. Then, $f(x) \leq s$, we have $f(y+x-y) \leq \max \{f(x)$, $0.5\} \leq s$. This implies that $(y+x-y)_{s}<f$.

(c) Now, let $x, y \in N$ and $s \in 0,1)$ such that $x_{s}<f$. Then, $f(x) \leq s$, we have $f(x y) \leq \max \{f(x), 0.5\} \leq s$, implies that $(x y)_{s}<f$. This completes the proof.

THEOREM 15: Any $(<,<\vee \Upsilon)$-fuzzy left ideal of $N$ such that $f(x)>0.5$ for all $x \in N$ is a $(<,<)$-fuzzy left ideal of $N$.

Proof: Condition (a) and condition $(b)$ of left ideal are proved in Theorem 14. (c) Now, let $x, y, a \in N$ and $s \in 0,1)$ such that $a_{s}<f$. Then, $f(a) \leq s$, we have $f(x(y+a)-x y) \leq \max \{f(a), 0.5\} \leq s, \quad$ implies that $(x(y+a)-x y)_{s}<f$. This completes the proof.

THEOREM 16: The union of any family of $(<,<\vee \Upsilon)$-fuzzy right ideals of $N$ is a $(<,<)$-fuzzy right ideal.

Proof: Let $\left\{f_{i}\right\}_{i \in \Lambda}$ be any family of $(<,<\vee \Upsilon)$-fuzzy right ideals of $N$ and $f=\cup_{i \in \Lambda} f_{i}={ }_{i \in \Lambda} f_{i}$.

Let $x, y \in N$. Then,

(a)

$$
\begin{aligned}
f(x-y)= & \left({ }_{i \in \wedge} f_{i}\right)(x-y) \\
& ={ }_{i \in \wedge}\left(f_{i}(x-y)\right)
\end{aligned}
$$

$$
\begin{aligned}
& \leq_{i \in \wedge}\left(\max \left\{f_{i}(x), f_{i}(y), 0.5\right\}\right) \\
& \left.=\max \left\{\left(_{i \in \wedge} f_{i}\right)(x),{ }_{i \in \wedge} f_{i}\right)(y), 0.5\right\} \\
f(x-y) \leq & \max \{f(x), f(y), 0.5\} .
\end{aligned}
$$

(b) Now,

$$
\begin{aligned}
& f(y+x-y)=\left({ }_{i \in \Lambda} f_{i}\right)(y+x-y) \\
& ==_{i \in \Lambda}\left(f_{i}(y+x-y)\right)
\end{aligned}
$$

(c) and,

$$
\begin{aligned}
& f(x y)=\left({ }_{i \in \Lambda} f_{i}\right)(x y)=_{i \in \Lambda}\left(f_{i}(x y)\right) \\
& \leq_{i \in \Lambda}\left(\max \left\{f_{i}(x), 0.5\right\}\right) \\
&=\max \left\{\left(_{i \in \Lambda} f_{i}\right)(x), 0.5\right\} \\
& f(x y) \leq \max \{f(x), 0.5\} .
\end{aligned}
$$

Hence, $f$ is a $(<,<)$-fuzzy right ideal of $N$. This completes the proof.

THEOREM 17: The union of any family of $(<,<\vee \Upsilon)$-fuzzy left ideals of $N$ is a $(<,<)$-fuzzy left ideal .

Proof: Let $\left\{f_{i}\right\}_{i \in \wedge}$ be any family of $(<,<\vee \Upsilon)$-fuzzy left ideals of $N$ and $f=\cup_{i \in \Lambda} f_{i}=_{i \in \Lambda} f_{i}$. Let $x, y, a \in N$. Then,

$$
\begin{aligned}
& f(x(y+a)-x y)=\left({ }_{i \in \Lambda} f_{i}\right)(x(y+a)-x y) \\
&= i_{i \in \Lambda}\left(f_{i}(x(y+a)-x y)\right. \\
& \leq_{i \in \Lambda}\left(\max \left\{f_{i}(a), 0.5\right\}\right) \\
&= \max \left\{\left(_{i \in \Lambda} f_{i}\right)(a), 0.5\right\} \\
& f(x(y+a)-x y) \leq \max \{f(a), 0.5\} .
\end{aligned}
$$

The remaining proof follows from Theorem 16.

For any fuzzy set $f$ in $N$ and $s \in 0,1)$, we denote $f^{s}=\left\{x \in N: x_{s}<f\right\}, \quad f_{s}=\left\{x \in N: x_{s} \Upsilon f\right\} \quad$ and $[f]_{s}=\left\{x \in N: x_{s}<\vee \Upsilon f\right\}$. 
Obviously, $[f]_{s}=f_{s} \cup f^{s}$, where $f^{s}, f_{s}$ and $[f]_{s}$ are called <-level set, $\Upsilon$-level set and $<\vee \Upsilon$-level set of $f$, respectively.

Lemma 6. Every fuzzy set $f$ in $N$ satisfies the following assertion:

(a) $[f]_{s}=f^{s}$ for $\left.s \in 0.5,1\right)$,

(b) $[f]_{s}=f_{s}$ for $\left.s \in 0,0.5\right)$.

\section{Proof:}

(a) Let $x \in[f]$, and $s \in 0.5,1)$. Then, $x_{s}<f$ or $x_{s} \Upsilon f$. If $x_{s}<f$, there is nothing to prove. Let $x_{s} \Upsilon f$ then, $f(x)+s<1$, which implies that $f(x)<s$. Thus $x \in f^{s}$. (b) Let $x \in[f]_{s}$ and $\left.s \in 0,0.5\right)$. Then $x_{s}<f$ or $x_{s} \Upsilon \mathrm{r} f$. If $x_{s} \Upsilon f$, there is nothing to prove. Let $x_{s}<f$, then $f(x)<s$, which implies that $f(x)+s<s+s<0.5+0.5=1$ Thus $x \in$ $f_{s}$. Which completes the proof.

Theorem 18. Let $f$ be a fuzzy set in $N$. Then, $f$ is a $(<,<\vee \Upsilon)$-fuzzy subnear-ring of $N$ if and only if $[f]_{s} \neq \varnothing$ is subnear-ring of $N$.

Proof: Assume that $f$ is a $(<,<\vee \Upsilon)$-fuzzy subnear-ring of $N$ and let $s \in 0,1)$ be such that $[f]_{s} \neq \varnothing$.

Let $x, y \in[f]_{s}$. Then, $f(x) \leq s$ or $f(x)+s<1$, and $f(y) \leq s$ or $f(y)+s<1$. We can consider four cases:

(i) $f(x) \leq s$ and $f(y) \leq s$,

(ii) $f(x) \leq s$ and $f(y)+s<1$,

(iii) $f(x)+s<1$ and $f(y) \leq s$,

(iv) $f(x)+s<1$ and $f(y)+s<1$.

For the first case, by Theorem $11(a)$, implies that

$$
\begin{aligned}
f(x-y) \leq & \max \{f(x), f(y), 0.5\} \\
& =\max \{s, 0.5\}=\left\{\begin{array}{l}
0.5 \text { if } s<0.5 \\
s \quad \text { if } s \geq 0.5
\end{array}\right.
\end{aligned}
$$

and so $f(x-y)+s<0.5+0.5=1$, i.e, $(x-y)_{s} \Upsilon f$, or $x-y \in f_{s}$. Therefore, $x-y \in f^{s} \cup f_{s}=[f]_{s}$. For the case (ii), assume that $s<0.5$. Then, $1-s>0.5$. If $\max \{f(x), 0.5\} \geq f(y)$, then $f(x-y) \leq \max \{f(x), 0.5\}<0.5$, and if $\max \{f(x), 0.5\}<f(y)$, then $f(x-y) \leq f(y) \leq 1-s$. Hence, $x-y \in f^{s} \cup f_{s}=[f]$, for $s<0.5$. Suppose that $s \geq 0.5$, then, $1-s \leq 0.5$. If $\max \{f(x), 0.5\} \geq f(y)$, then $f(x-y) \leq \max \{f(x), 0.5\} \leq s$, and if $\max \{f(x), 0.5\}<f(y)$, then $f(x-y) \leq f(y)<1-s$. Thus, $x-y \in f^{s} \cup f_{s}=[f]_{s}$ for $s \geq 0.5$. We have similar result for the case (iii). For final case, $s<0.5$, then $1-s>0.5$. Hence,

$$
\begin{gathered}
f(x-y) \leq \max \{f(x), f(y), 0.5\} \\
=\left\{\begin{array}{cc}
0.5<1-s \quad \text { if } \max \{f(x), f(y)\} \leq 0.5, \\
\max \{f(x), f(y)\}<1-s \text { if } \max \{f(x), f(y)\}>0.5,
\end{array}\right.
\end{gathered}
$$

and so $(x-y) \in f_{s} \subseteq[f]_{s}$. If $s \geq 0.5$, then $1-s \leq 0.5$. Thus,

$$
\begin{gathered}
f(x-y) \leq \max \{f(x), f(y), 0.5\} \\
=\left\{\begin{array}{cc}
0.5 \leq s \quad \text { if } \max \{f(x), f(y)\} \leq 0.5, \\
\max \{f(x), f(y)\}<1-s \text { if } \max \{f(x), f(y)\}>0.5,
\end{array}\right.
\end{gathered}
$$

which implies that $x-y \in f^{s} \cup f_{s}=[f]$. Similary we can prove that $x y \in[f]_{s}$. Therefore $[f]_{s} \neq \varnothing$ is subnearring of $N$.

Conversely, suppose that $f$ is a fuzzy set in $N$ such that $[f]_{s}$ is a sub-near-ring of $N$. Suppose that $f$ is not a $(<,<\vee \Upsilon)$-fuzzy subnear-ring of $N$. Then, there exist $x, y \in N$ such that $f(x-y)>\max \{f(x), f(y), 0.5\}$. Let $s=\frac{1}{2}[f(x-y)+\max \{f(x), f(y), 0.5\}]$. Then, $f(x-y)>$ $s>\max \{f(x), f(y), 0.5\}$. This implies that $x, y \in[f]_{s}$ and $(x-y) \notin[f]_{s}$, which is a contradiction. Therefore, we have $f(x-y) \leq \max \{f(x), f(y), 0.5\}$, for all $x, y \in N$. Similarly, we can show that $f(x y) \leq \max \{f(x), f(y), 0.5\}$, for all $x, y \in N$. Therfore $f$ is a $(<,<\vee \Upsilon)$-fuzzy subnear-ring of $N$.

Theorem 19. Let $f$ be a fuzzy set in $N$. Then, fis a $(<,<\vee \Upsilon)$ fuzzy right ideal of $N$ if and only if $[f]_{s} \neq \varnothing$ is a right ideal of $N$.

Proof: Assume that $f$ is a $(<,<\vee \Upsilon)$-fuzzy right ideal of $N$ and let $s \in 0,1)$ be such that $[f]_{s} \neq \varnothing$

(a) Let $x, y \in[f]_{s}$. Then, $\left.x-y \in f\right]_{s}$ proved in Theorem 18 . (b) Now, let $x \in[f]_{s}$ and $y \in N$. Then, $f(x) \leq s$ or $f(x)+s<1$. Assume that $f(x) \leq s$. Then by Theorem $11(b)$,

$$
\begin{array}{r}
f(y+x-y) \leq \max \{f(x), 0.5\} \leq \max \{s, 0.5\} \\
=\left\{\begin{array}{cc}
s, & \text { if } s \geq 0.5, \\
0.5<1-s, & \text { if } s<0.5,
\end{array}\right.
\end{array}
$$

so that $y+x-y \in f^{s} \cup f_{s}=[f]_{s}$. Suppose that $f(x)+s<1$, then

$$
\begin{aligned}
f(y+x-y) \leq & \max \{f(x), 0.5\} \leq \max \{1-s, 0.5\} \\
= & \begin{cases}0.5, & \text { if } s \geq 0.5, \\
1-s, & \text { if } s<0.5,\end{cases}
\end{aligned}
$$


and thus $y+x-y \in f_{s} \cup f_{s}=[f]_{s}$.

(c) Now, let $x \in[f]$, and $y \in N$. Then, $f(x) \leq s$ or $f(x)+s<1$. Assume that $f(x) \leq s$. Then by Theorem $11(c)$,

$$
\begin{aligned}
f(x y) \leq & \max \{f(x), 0.5\} \geq \max \{s, 0.5\} \\
& = \begin{cases}s, & \text { if } s \geq 0.5, \\
0.5<1-s, & \text { if } s<0.5,\end{cases}
\end{aligned}
$$

so that $x y \in f^{s} \cup f_{s}=[f]_{s}$. Suppose that $f(x)+s<1$, then

$$
\begin{aligned}
f(x y) & \leq \max \{f(x), 0.5\}<\max \{1-s, 0.5\} \\
& = \begin{cases}0.5 & \text { if } s \geq 0.5, \\
1-s, & \text { if } s<0.5,\end{cases}
\end{aligned}
$$

and thus $x y \in f^{s} \cup f_{s}=[f]_{s}$. Therefore $[f]_{s}$ is a right ideal of $N$.

Conversely, suppose that $f$ is a fuzzy set in $N$ such that $[f]_{\mathrm{s}}$ is a right ideal of $N$. (a) Let $x, y \in N$, then $f(x-y) \leq \max \{f(x), f(y), 0.5\}$, proved in Theorem 18 . (b) Let $x, y \in N$ such that $f(y+x-y)>\max \{f(x), 0.5\}$.

Let $s=\frac{1}{2}[f(y+x-y)+\max \{f(x), 0.5\}]$. Then, $f(y+x-$ $y)>s>\max \{f(x), 0.5\}$. This implies that $x \in[f]_{s}$ and $(y+x-y) \notin[f]_{s}$, which is a contradiction. Therefore, $f(y+x-y) \leq \max \{f(x), 0.5\}$, for all $x, y \in N$. (c) Let $x, y \in N$ such that $f(x y)>\max \{f(x), 0.5\}$. Let $s=\frac{1}{2}[f(x y)+\max \{f(x), 0.5\}]$. Then, $f(x y)>s>\max \{f$ $(x), 0.5\}$. This implies that $x \in[f]_{s}$ and $(x y) \notin[f]_{s}$, which is a contradiction. Therefore, $f(x y) \leq \max \{f(x)$, $0.5\}$, for all $x, y \in N$. Hence, $f$ is a $(<,<\vee \Upsilon)$-fuzzy right ideal of $N$. This completes the proof.

Theorem 20. Let fbe a fuzzy set in $N$. Then, fis a $(<,<\vee \Upsilon)$ fuzzy left ideal of $N$ if and only if $[f]_{s} \neq \varnothing$ is a left ideal of $N$.

Proof: Assume that $f$ is a $(<,<\vee \Upsilon)$-fuzzy left ideal of $N$ and let $s \in 0,1)$ be such that $[f]_{s} \neq \varnothing$. (a) Let $x, y \in[f]_{s}$. Then, $\left.x-y \in f\right]_{s}$ proved in Theorem 18 .

(b) Now, let $x \in[f]_{s}$ and $y \in N$. Then, $\left.x y \in f\right]_{s}$ proved in Theorem 19. (c) Now, let $a \in[f]_{s}$ and $x, y \in N$. Then, $f(a) \leq s$ or $f(a)+s<1$. Assume that $f(a) \leq s$. Then by Theorem $12(c)$,

$$
\begin{array}{r}
f(x(y+a)-x y) \leq \max \{f(a), 0.5\} \leq \max \{s, 0.5\} \\
= \begin{cases}s, & \text { if } s \geq 0.5, \\
0.5<1-s, & \text { if } s<0.5,\end{cases}
\end{array}
$$

so that $x(y+a)-x y \in f^{s} \cup f_{s}=[f]_{s}$. Suppose that $f(a)+s<1$, then

$$
\begin{gathered}
f(x(y+a)-x y) \leq \max \{f(a), 0.5\}<\max \{1-s, 0.5\} \\
= \begin{cases}0.5, & \text { if } s \geq 0.5, \\
1-s, & \text { if } s<0.5,\end{cases}
\end{gathered}
$$

and thus $x(y+a)-x y \in f^{s} \cup f_{s}=[f]_{s}$. Consequently, $[f]_{\mathrm{s}}$ is a left ideal of $N$.

Conversely, suppose that $f$ is a fuzzy set in $N$ such that $[f]$ is an ideal of $N$. Let $x, y \in N$. Then, $(a) f(x-y) \leq \max$ $\{f(x), f(y), 0.5\}$, by Theorem 18. (b) $f(y+x-y) \leq$ $\max \{f(x), 0.5\}$, by Theorem 19. (c) Let $x, y, a \in N$ such that $f(x(y+a)-x y)>\max \{f(a), 0.5\}$.

Let $s=\frac{1}{2}[f(x(y+a)-x y)+\max \{f(a), 0.5\}] . \quad$ Then, $f(x(y+a)-x y)>s>\max \{f(a), 0.5\}$. This implies that $x \in[f]_{s}$ and $x(y+a)-x y \notin[f]_{s}$, which is a contradiction. Therefore, $f(x(y+a)-x y) \leq \max \{f(a), 0.5\}$, for all $x, y, a \in N$. $f$. Hence, $f$ is a $(<,<\vee \Upsilon)$-fuzzy left ideal of $N$. This completes the proof.

Theorem 21. Let $f$ be a fuzzy set in $N$. Then $f$ is a $(<,<\vee \Upsilon)$ -fuzzy subnear-ring of $N$ if and only if $f^{s} \neq \varnothing$ is subnear-ring ideal of $N$ for all $s \in 0.5,1)$.

Proof: Let $f$ is a $(<,<\vee \Upsilon)$-fuzzy subnear-ring of $N$, then by Theorem 18, $[f]_{s}$ is a subnear-ring of $N$. Also by Lemma $6,[f]_{s}=f^{s}$. Thus $f^{s} \neq \varnothing$ is a subnear-ring of $N$.

Conversly, let $f^{s} \neq \varnothing$ is subnear-ring of $N$. Then by Lemma 6 and Theorem 18, we have that $f$ is a $(<,<)$ fuzzy subnear-ring of $N$.

Theorem 22. Let $f$ be a fuzzy set in $N$. Then $f$ is a $(<,<\vee \Upsilon)$-fuzzy right ideal of $N$ if and only if $f^{s} \neq \varnothing$ is right ideal of $N$ for all $s \in 0.5,1)$.

Proof: Let $f$ is a $(<,<\vee \Upsilon)$-fuzzy right ideal of $N$, then by Theorem 19, $[f]_{s}$ is a right ideal of $N$. Also by Lemma $6,[f]_{s}=f^{s}$. Thus $f^{s} \neq \varnothing$ is a right ideal of $N$.

Conversly, let $f^{s} \neq \varnothing$ is a right ideal of $N$. Then by Lemma 6 and Theorem 19, we have that $f$ is a $(<,<\vee \Upsilon)$ fuzzy right ideal of $N$. 
Theorem 23. Let $f$ be a fuzzy set in $N$. Then $f$ is a $(<,<\vee \Upsilon)$-fuzzy left ideal of $N$ if and only if $f^{s} \neq \varnothing$ is a left ideal of $N$ for all $s \in 0.5,1)$.

Proof: Let $f$ is a $(<,<\vee \Upsilon)$-fuzzy left ideal of $N$, then by Theorem 20, $[f]_{s}$ is a left ideal of $N$. Also by Lemma 6 , $[f]_{s}=f^{s}$. Thus $f^{s} \neq \varnothing$ is a left ideal of $N$.

Conversly, let $f^{s} \neq \varnothing$ is a left ideal of $N$. Then by Lemma 6 and Theorem 20, we have that $f$ is a $(<,<\vee \Upsilon)$ fuzzy left ideal of $N$.

Corollary 1. For a fuzzy set $f$ in $N$, the following are equivalent

(a) $[f]_{s}=\left\{x \in N: x_{s},<\vee \Upsilon f\right\}$ for $\left.s \in 0,1\right)$ is a sub-near-ring of $N$,

(b) Conditions $(a)$ and $(b)$ of Theorem 10.

Proof: $(a) \Rightarrow(b)$, let $[f]_{s}=\left\{x \in N: x_{s},<\vee \Upsilon f\right\}$ for $s \in 0,1)$ is a sub-near-ring of $N$. Then by Theorem 19, $f$ is a $(<,<\vee \Upsilon)$-fuzzy sub-near-ring of $N$. Similarly, we can show that $(b) \Rightarrow(a)$ by using Theorem 10 and Theorem 19.

Corollary 2. For a fuzzy set $f$ in $N$, the following are equivalent

(a) $[f]_{s}=\left\{x \in N: x_{s}<\vee \Upsilon f\right\}$ for $\left.s \in 0,1\right)$ is a right ideal of $N$,

(b) Conditions (a), (b) and (c) of Theorem 11.

Proof: $(a) \Rightarrow(b)$, let $[f]_{s}=\left\{x \in N: x_{s},<\vee \Upsilon f\right\}$ for $s \in$ $0,1)$ is a right ideal of $N$. Then by Theorem $19, f$ is a $(<,<\vee \Upsilon)$-fuzzy right ideal of $N$. Similarly, we can show that $(b) \Rightarrow(a)$ by using Theorem 11 and Theorem 19.

Corollary 3. For a fuzzy set $f$ in $N$, the following are equivalent

(a) $[f]_{s}=\left\{x \in N: x_{s}<\vee \Upsilon f\right\}$ for $\left.s \in 0,1\right)$ is a left ideal of $N$,

(b) Conditions $(a),(b)$ and $(c)$ of Theorem 12.

Proof: $\quad(a) \Rightarrow(b)$, let $[f]_{s}=\left\{x \in N: x_{s}<\vee \Upsilon f\right\}$ for $s \in 0,1)$ is a left ideal of $N$. Then by Theorem $20, f$ is a $(<,<\vee \Upsilon)$-fuzzy left ideal of $N$. Similarly, we can show that $(b) \Rightarrow(a)$ by using Theorem 12 and Theorem 20 .

\section{Prime $(<,<\vee r)$-Fuzzy Ideals}

In this section, we describe semiprime and prime $(<,<\vee)$-fuzzy ideals of near-rings and investigate some properties of these ideals.
Definition 12. $A(<,<\vee \Upsilon)$-fuzzy ideal $f$ of $N$ is called semiprime if for all $x \in N$ and $s \in 0,1),\left(x_{s}\right)^{2}<f$ implies that $x_{s}<\vee \Upsilon f$. A $(<,<\vee \Upsilon)$-fuzzy ideal $f$ of $N$ is called prime if for all $x, y \in N$ and $s \in 0,1),(x y)_{s}<f$ implies that $x_{s}<\vee \Upsilon f$ or $y_{s}<\vee \Upsilon f$

TheOREM 24. Let $f$ be a prime $(<,<\vee \Upsilon)$-fuzzy ideal of $N$. Then, the set $N_{1}=\{x \in N: f(x)<1\} \neq \varnothing$ is a prime ideal of $N$.

Proof: The proof follows from Theorem 5.

Corollary 4. Let fbe a semiprime $(<,<\vee \Upsilon)$-fuzzy ideal of $N$. Then, the set $N_{1}=\{x \in N: f(x)<1\} \neq \varnothing$ is a semiprime ideal of $N$.

Proof: Straightforward.

THEOREM 25. Let $P$ be a prime ideal of $N$ and $f$ a fuzzy set of $N$ such that

(i) $f(x) \leq 0.5$ for $x \in P$,

(ii) $f(x)=1$ otherwise.

Then, $f$ is a prime $(<,<\vee \Upsilon)$-fuzzy ideal of $N$.

Proof: Let $P$ is a prime ideal of $N$. Then, by Theorem $8, f$ is an $(<,<\vee \Upsilon)$-fuzzy ideal of $N$. Now, let $x, y \in N$ and $s \in 0,1)$ such that $(x y)_{s}<f$. Then, $f(x y) \leq s$, this implies that $(x y) \in P$. Since, $P$ is a prime, so $x \in P$ or $y \in P$, which implies that $f(x) \leq 0.5$ or $f(y) \leq 0.5$. If $s \geq 0.5$, then $f(x) \leq 0.5 \leq s$ or $f(y) \leq 0.5 \leq s$, which implies that $x_{s}<f$ or $y_{s}<f$. Now, if $s<0.5$, then $f(x)+s<0.5+0.5=1$ or $f(y)+s<0.5+0.5=1$,

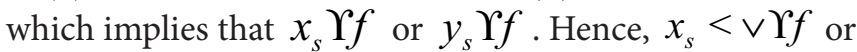
$y_{s}<\vee \Upsilon f$. Therefore, $f$ is a prime $(<,<\vee \Upsilon)$-fuzzy ideal of $N$. This completes the proof.

Corollary 5. Let $P$ be a semiprime ideal of $N$ and $f a$ fuzzy set of $N$ such that

(i) $f(x) \leq 0.5$ for $x \in P$,

(ii) $f(x)=1$ otherwise.

Then, $f$ is a semiprime $(<,<\vee \Upsilon)$-fuzzy ideal of $N$.

Proof: Straightforward.

TheORem 26. $A(<,<\vee \Upsilon)$-fuzzy ideal $f$ of $N$ is prime if and only if $\min \{f(x), f(y)\} \leq \max \{f(x y), 0.5\}$, for all $x, y \in N$.

Proof: Let $f$ be a prime $(<,<\vee \Upsilon)$-fuzzy ideal of $N$. Suppose that there exist some $x, y \in N$ such that $\min \{f(x), f(y)\}>\max \{f(x y), 0.5\}$. Then, $\min \{f(x), f(y)\}$ $>s>\max \{f(x y), 0.5\}$, for some $s \in 0,1)$. This means that 
$(x y)_{s}<f$ but $x_{s} \overline{<} f$ or $y_{s} \overline{<} f$, which is a contradiction. Hence, $\min \{f(x), f(y)\} \leq \max \{f(x y), 0.5\}$, for all $x, y \in N$.

Conversely, assume that the following hold for all $x, y \in N, \min \{f(x), f(y)\} \leq \max \{f(x y), 0.5\}$. Let $(x y)_{s}<f$. Then, we see that $\min \{f(x), f(y)\} \leq \max \{s, 0.5\}$. If $s \geq 0.5$, then either $f(x) \leq s$ or $f(y) \leq s$, this implies that $x_{s}<f$ or $y_{s}<f$. If $s<0.5$, then either $f(x)+s<0.5+0.5=1$ or $f(y)+s<0.5+0.5=1$, this implies that $x_{s} \Upsilon f$ or $y_{s} \Upsilon f$. Hence $x_{s}<\vee \Upsilon f$ or $y_{s}<\vee \Upsilon f$.

Corollary 6. $a(<,<\vee \Upsilon)$-fuzzy ideal $f$ of $N$ is semiprime if and only if $f(x) \leq \max \left\{f\left(x^{2}\right), 0.5\right\}$, for all $x \in N$.

Proof: Straightforward.

THeOReM 27. Union of any family of prime $(<,<\vee \Upsilon)$ fuzzy ideals of $N$ is a prime $(<,<\vee \Upsilon)$-fuzzy ideal of $N$.

Proof: By Theorem 16, $f=\cup_{i \in \Lambda} f_{i}={ }_{i \in \Lambda} f_{i}$ is a $(<,<\vee \Upsilon)$ fuzzy ideal of $N$. Now, let $x, y \in N$. Then, we have $\min \{f(x), f(y)\}=\min \left\{\left({ }_{i \in \Lambda} f_{i}\right)(x),\left({ }_{i \in \Lambda} f_{i}\right)(y)\right\}$

$$
\begin{aligned}
& =\min \left\{{ }_{i \in \Lambda}\left(f_{i}(x)\right)_{,_{i \in \Lambda}}\left(f_{i}(y)\right)\right\} \\
& \leq \max \left\{\left({ }_{i \in \Lambda} f_{i}\right)(x),\left(_{i \in \Lambda} f_{i}\right)(y), 0.5\right\} \\
& =\max \{f(x), f(y), 0.5\}
\end{aligned}
$$

$\min \{f(x), f(y)\} \leq \max \{f(x), f(y), 0.5\}$.

Hence, by Theorem 26, $f$ is a prime $(<,<\vee \Upsilon)$-fuzzy ideal of $N$.

COROLlaRY 7. Union of any family of semiprime $(<,<\vee \Upsilon)$ fuzzy ideals of $N$ is a semiprime $(<,<\vee \Upsilon)$-fuzzy ideal of $N$.

Proof: Straightforward.

TheOREM 28. $A(<,<\vee \Upsilon)$-fuzzy ideal $f$ of $N$ is prime if and only if for $0.5 \leq s<1, f^{s} \neq \varnothing$ is a prime ideal of $N$.

Proof: Let $f$ be a prime $(<,<\vee \Upsilon)$-fuzzy ideal of $N$ and let $0 \leq s<0.5$. Then, by Theorem 21 , each non-empty $f^{s}$ is an ideal of $N$. Let $x, y \in N$ be such that $x y \in f^{s}$. Then, $(x y)_{s}<f$. By Theorem 26, we have $x \in f^{s}$ or $y \in f^{s}$. Hence $f^{s}$ is a prime ideal of $N$.

Conversely, assume that $f^{s}$ is a prime ideal of $N$ for $0 \leq s<0.5$. Then, by Theorem $21, f$ is a $(<,<\vee \Upsilon)_{\text {-fuzzy }}$ ideal of $N$. Let $x, y \in N$ be such that $(x y)_{s}<f$ and $s \geq 0.5$. Then, $x y \in f^{s}$. Since $f^{s}$ is a prime, so either $x \in f^{s}$ or $y \in f^{s}$, this implies that $x_{s}<f$ or $y_{s}<f$. Therefore, $x_{s}<\vee \Upsilon f$ or $y_{s}<\vee \Upsilon f$. Hence, $f$ is a prime $(<,<\vee \Upsilon)_{\text {- }}$ fuzzy ideal of $N$.
Proposition 2. $A(<,<\vee \Upsilon)$-fuzzy ideal $f$ of $N$ is prime if and only if for $0 \leq s<0.5, f_{s} \neq \varnothing$ is a prime ideal of $N$.

Proof: Let $f$ be a prime $(<,<\vee \Upsilon)$-fuzzy ideal of $N$ and let $0 \leq s<0.5$. Then, by Theorem 21, each non-empty $f_{s}$ is an ideal of $N$. Let $x, y \in N$ be such that $x y \in f^{s}$. Then, $(x y)_{s} \Upsilon f$. By Theorem 26, we have $x \in f_{s}$ or $y \in f_{s}$. Hence $f_{s}$ is a prime ideal of $N$.

Conversely, assume that $f_{s}$ is a prime ideal of $N$ for $0 \leq s<0.5$. Then, by Theorem $21, f$ is a $(<,<\vee \Upsilon)$-fuzzy ideal of $N$. Let $x, y \in N$ be such that $(x y)_{s} \Upsilon f$. Then, $x y \in f_{s}$. Since $f_{s}$ is a prime, so either $x \in f_{s}$ or $y \in f_{s}$, this implies that $x_{s} \Upsilon f$ or $y_{s} \Upsilon f$. Hence, $f$ is a prime $(<,<\vee \Upsilon)$-fuzzy ideal of $N$.

Corollary 8. A $(<,<\vee \Upsilon)$-fuzzy ideal $f$ of $N$ is semiprime if and only if for $0.5 \leq s<1, f^{s} \neq \varnothing$ is a semiprime ideal of $N$.

Proof: Straightforward.

COROLLARY 9. $A(<,<\vee \Upsilon)$-fuzzy ideal $f$ of $N$ is semiprime if and only if for $0 \leq s<0.5, f_{s} \neq \varnothing$ is a semiprime ideal of $N$.

Proof: Straightforward.

THEOREM 29. A fuzzy set $f$ of $N$ is a prime (semiprime) $(<,<\vee \Upsilon)$-fuzzy ideal if and only if for $0 \leq s<1,[f]_{s}$ is a prime (semiprime) ideal of $N$.

Proof: Proof follows from Theorem 19 and Theorem 28.

\section{Conclusion}

In the study of the structure of an anti-fuzzy algebraic system, we notice that anti-fuzzy ideals with special properties always play an important role. In this paper, we define $(<,<\vee \Upsilon)$-fuzzy subnear-ring(left, right two-sided) ideals of near-rings and investigated the relationship among these generalized anti-fuzzy subnear-ring (left, right) ideals of near-rings. Finally, we define prime(semiprime) $(<,<\vee \Upsilon)$-fuzzy ideals of near-rings and investigated some important results. Some characterization theorems of prime(semiprime) $(<,<\vee \Upsilon)$-fuzzy ideals of near-rings are obtained. We hope that the research along this direction can be continued, and in fact, this work would serve as a foundation for further study of the theory of near-ring, it will be necessary to carry out more theoretical research to establish a general framework for the practical application.

In our future study of anti-fuzzy structure of near-rings, may be the following topics should be considered: (1): To 
focus on other types of near-ring with similar nature and study their mutual relationships; (2): To establish an antifuzzy spectrum of near-ring; (3): To consider the structure of quotient near-ring by using $(<,<\vee \Upsilon)$-fuzzy subnearring (left, right) ideals; (4): To describe fuzzy soft near-ring and its applications; (5): To describe anti-fuzzy soft nearrings and its applications in informations sciences and general systems.

\section{References}

1. Dickson, and Leonard E (1905). Definitions of a group and a field by independent postulates, Transactions of the American Mathematical Society, vol 6, No. 2, 198-204.

2. Zadeh L A (1965). Fuzzy sets, Information and Control, vol 8(3), 338-353.

3. Abou-Zaid S (1991). On fuzzy subnear-ring and ideals, Fuzzy Sets and System, vol 44(1), 139-146.

4. Hong S M, Jun Y B et al. (1996). Fuzzy ideals of near-rings, Bulletin of the Korean Mathematical Society, vol 35, 455-464.

5. Kim S D, and Kim H S (1996). On fuzzy ideals of near-rings, Bulletin of the Korean Mathematical Society, vol 33, No. 4, 593-601.

6. Biswas R (1990). Fuzzy subgroups and anti-fuzzy subroups, Fuzzy Sets and Sys, vol 35(1), 121-124.

7. Kim K H, and Jun Y B (1999). Anti fuzy R-subgroups of near-rings, Scientiae Mathematicae, vol 2, No. 2, 147-153.

8. Kim K H, Jun Y B et al. (2005). On anti fuzy ideals in nearrings, Iranian Journal of Fuzzy Systems, vol 2, No. 2, 71-80.
9. Shabir M, and Rehman N (2011). Characterizations of ternary semigroups by their anti-fuzzy ideals, Annals of Fuzzy Mathematics and Informatcs, vol 2, No. 2, 227-238.

10. Jun Y B, Khan A et al. (2010). Generalized anti-fuzzy biideals in ordered semigroups, Lobachevskii Journal of Mathematics, vol 31, No. 1, 65-76.

11. Akram M, and Dar K H (2007). On anti-fuzzy left $h$-ideals in hemirings, International Mathematical Forum, vol 2, No. 46, 2295-2304.

12. Hong S M, and Jun Y B (1998). Anti-fuzzy ideals in BCK-algebras, Kyungpook Mathematical Journal, vol 38, 145-150.

13. Jeong W K (1999), On anti-fuzzy prime ideals in BCKalgebras, Journal of the Chungcheong Mathematical Society, vol 12, 15-21.

14. Shabir M, and Nawas Y (2009). Semigroups characterizatised by their anti-fuzzy ideals, Journal of Advanced Research in Pure Mathematics, vol 3, 42-59.

15. Khan M, Iqbal M F et al. (2010). On anti-fuzzy Ideals in Left Almost Semigroups, Journal of Mathematics Research, vol 2, No. 3, 203-210.

16. Saeid A B, and Jun Y B (2008). Redefind fuzzy subalgebras of BCK/BCI-algebras, Iranian Journal of Fuzzy Systems, vol $5(2), 63-70$.

17. Pilz G (1977). Near-rings the theory and its Applications, North-Holland Publishing Com.

18. Narayanan $\mathrm{Al}$, and Manikantan $\mathrm{T}$ (2005). $(\in, \in \vee q)$ fuzzy subnear-rings and $(\in, \in \vee q)$-fuzzy ideals of nearrings, Journal of Applied Mathematics and Computing, vol $18,419-430$. 